\title{
Wheat desensitization treatment in patients with gluten sensitivity
}

\author{
Eleonora Nucera ${ }^{1}$, Arianna Aruanno ${ }^{1}$, Gianluca laniro², Giovanni Cammarota ${ }^{2}$, Antonio Gasbarrini², Domenico Schiavino ${ }^{1}$
}

${ }^{1}$ Allergy Unit, Fondazione Policlinico Universitario A. Gemelli IRCSS - Università Cattolica del Sacro Cuore, Roma, Italy ${ }^{2}$ Department of Internal Medicine, Gastroenterology and Hepatology, Fondazione Policlinico Universitario A. Gemelli IRCSS - Università Cattolica del Sacro Cuore, Roma, Italy

Adv Dermatol Allergol 2018; XXXV (3): 320-322

DOI: https://doi.org/10.5114/ada.2018.76229

Gluten is the main structural protein of wheat and other cereals (barley, rye, etc.) and is one of the principal dietary components for most of the world population since the birth of agriculture, playing a key role in the development of human beings. On the other hand, this evolutionary trick has led to several diseases related to gluten exposure, the best studied of which is celiac disease (CD) and wheat allergy (WA). However, some individuals complain of gastrointestinal or extra-intestinal symptoms linked to the ingestion of gluten and disappearing with gluten withdrawal, even without any histological or serologic evidence of celiac disease (CD) or wheat allergy (FA). In most cases these patients consult a large number of physicians seeking the diagnosis of CD, but very often they are considered to be suffering from irritable bowel syndrome (IBS) only.

According to a recently developed consensus, the term "non-celiac gluten sensitivity" (NCGS) has been suggested for this condition, relating to "one or more of a variety of immunological, morphological or symptomatic manifestations that are induced by the ingestion of gluten in people in whom CD has been excluded" [1] and the diagnosis is possible only after exclusion of the celiac disease, food allergies or food-related disorders and the symptomatic response to a change in the diet [2]. The diagnostic "gold standard" for gluten sensitivity ought to be the double-blind placebo-controlled (DBPC) challenge, but it is a quite dangerous and time-consuming method and is therefore rarely used [3].

There are no objective findings or markers to support a diagnosis of gluten sensitivity and its pathogenesis remains obscure, although the hypothesis is based on a stimulation of the innate immune system by the gliadin. Another theory suggests that the gliadin induces a direct cytotoxic effect on enterocytes [4, 5]. However, a role for gluten proteins as the sole trigger of the associated symptoms remains to be established.
Although the patients with gluten sensitivity have a beneficial effect on their symptoms with the glutenfree diet, the same new diet reduces enormously their quality of life. For these reasons it is important not only to provide the understanding of the NCGS pathogenesis, but also how to manage this disease to enhance the quality of life of the patients.

Therefore, we present a case report about a girl with NCGS who underwent successful wheat desensitization treatment.

We report the case of a 27-year-old girl with abdominal pain, diarrhea, oral itching and throat tightness after the ingestion of wheat products. The girl had wheat allergological tests done after a previous gastroenterological evaluation to fulfill the criteria recently proposed on "gluten sensitivity" $[2,6]$.

She had anti-gliadin (AGA) IgA and IgG antibodies, anti-transglutaminase (anti-tTG) and anti-endomysial (EmA) IgA antibodies, esophagogastroduodenoscopy with biopsy, HLA molecular typing for celiac disease and lactulose breath test in the gastroenterological screening and wheat skin prick test, specific IgE assay and basophil activation test for the allergological evaluation.

At the end of the diagnostic tests, the patient met all the following inclusion criteria:

- IBS-like symptoms defined according to the Rome II criteria [7];

- Negative serum anti-tTG and EmA IgA antibodies;

- Negative duodenal histology (absence of intestinal villous atrophy);

- Negative wheat skin prick test and specific IgE assay [8];

- Resolution of the symptoms on gluten-free diet and reappearance on DBPC wheat challenge [9].

Since the girl presented positive HLA DQ2 haplotype and lactulose breath test, she had antibiotic and probiotic therapy for small intestinal bacterial overgrowth with a subsequent negative breath test. All allergological tests

Address for correspondence: Dr. Arianna Aruanno, Allergy Unit, Università Cattolica del Sacro Cuore, Policlinico “A. Gemelli”, Largo F. Vito, 1 - 00168 Rome, Italy, phone: +3906 30155896, fax: +3906 30156999, e-mail: aarianna@hotmail.it Received: 22.09.2016, accepted: 26.03.2017. 
were negative and, as she continued to present symptoms, the patient began to avoid all products containing wheat on her own with complete disappearance of clinical manifestations.

Therefore, we did a DBPC challenge (Table 1) with wheat and she presented a systemic reaction (abdominal pains, nausea and oral itching with mucosal hyperemia) after the ingestion of $15 \mathrm{ml}$ of this food.

At this point the girl underwent a wheat desensitization treatment (Table 2) and after 21 months she was able to eat about 150 gr of wheat without any adverse reaction, starting a free dietary regimen.

In the clinical practice, an emerging problem is how to manage patients who experience symptoms after wheat ingestion in the absence of the main symptoms of CD or food allergy. These subjects often start glutenfree diet on their own with a complete benefit on manifestations.

The elimination diet itself reduces quality of life, leading to social, economic, psychological problems.

Moreover, we do not know the possible complications or how strictly or for how long the diet should be adhered to. So gluten sensitivity needs further investigations before gluten-free foods are promoted as a remedy on a large scale.

In this case report we have tried to manage our patient with the same therapeutic method we use in allergic patients and we obtained good results allowing her to enlarge the diet without any restrictions.

Moreover, as in allergic diseases, in NCGS we found a very high frequency of self-reported wheat intolerance that makes the patients choose the elimination diet. The DBPC challenge is widely used to confirm the diagnosis of food hypersensitivity and therefore could be a diagnostic marker for NCGS, able to overcome the emotional component that seems to be pivotal in these patients. In this case report we have shown how the wheat DBPC test could be the "gold standard" method also to make the diagnosis in these subjects, excluding the subjec-
Table 1. Wheat DBPC challenge (boiled semolina $40 \mathrm{mg} / \mathrm{ml}$ )

\begin{tabular}{lc}
\hline Day 1: & \\
\hline Dilution $1: 100$ & $0.1 \mathrm{ml}$ \\
\hline Dilution $1: 100$ & $0.5 \mathrm{ml}$ \\
\hline Dilution $1: 100$ & $1 \mathrm{ml}$ \\
\hline Dilution $1: 10$ & $0.2 \mathrm{ml}$ \\
\hline Dilution $1: 10$ & $0.3 \mathrm{ml}$ \\
\hline Dilution $1: 10$ & $0.4 \mathrm{ml}$ \\
\hline Day 2: & \\
\hline Pure solution & $0.2 \mathrm{ml}$ \\
\hline Pure solution & $0.5 \mathrm{ml}$ \\
\hline Pure solution & $1 \mathrm{ml}$ \\
\hline Pure solution & $2 \mathrm{ml}$ \\
\hline Pure solution & $5 \mathrm{ml}$ \\
\hline Pure solution & $10 \mathrm{ml}$ \\
\hline
\end{tabular}

tive component that seems to prevail in this disease. Carroccio et al. [3] have reached the same conclusions and demonstrated that DBPC challenge confirmed the diagnosis of NCGS in a relevant percentage of patients. Although DBPC test seems to have a high diagnostic accuracy, its use is limited to the research setting because it is expensive and time-consuming [10]; therefore, a reasonable approach in clinical practice would be to perform cheaper and easier single-blind gluten challenge tests.

Although this is only a case report, our results represent a new approach in research on NCGS and could provide a future perspective of the study for its management.

\section{Acknowledgments}

Dr. Nucera Eleonora and Dr. Aruanno Arianna have equally participated in the realization of the present study.

Table 2. Wheat desensitization treatment

\begin{tabular}{|c|c|c|c|c|c|}
\hline Wheat & Days & Dilution & $\begin{array}{l}\text { Starting dose } \\
\text { (once a day) }\end{array}$ & $\begin{array}{c}\text { Final dose } \\
\text { (3 times a day) }\end{array}$ & Daily dose [g] \\
\hline \multirow{6}{*}{$\begin{array}{l}\text { Semolina } \\
(0.02 \mathrm{~g} / \mathrm{ml})\end{array}$} & $1-18$ & $10^{-6}$ & $1 \mathrm{ml}$ & $10 \mathrm{ml}$ & $0.6 \times 10^{-6}$ \\
\hline & $19-30$ & $10^{-4}$ & $1 \mathrm{ml}$ & $10 \mathrm{ml}$ & $0.6 \times 10^{-4}$ \\
\hline & $31-39$ & $10^{-3}$ & $3 \mathrm{ml}$ & $10 \mathrm{ml}$ & $0.6 \times 10^{-3}$ \\
\hline & $40-48$ & $10^{-2}$ & $3 \mathrm{ml}$ & $10 \mathrm{ml}$ & $0.6 \times 10^{-2}$ \\
\hline & $49-57$ & $10^{-1}$ & $3 \mathrm{ml}$ & $10 \mathrm{ml}$ & 0.06 \\
\hline & $58-120$ & Pure solution & $3 \mathrm{ml}$ & $75 \mathrm{ml}$ & 4.5 \\
\hline \multirow{2}{*}{$\begin{array}{l}\text { Pasta } \\
\text { (1 spagh/portion = } 1.2 \mathrm{~g})\end{array}$} & $121-144$ & Pure solution & 1 spaghetti & 4.5 spaghetti & 16.2 \\
\hline & $145-215$ & Pure solution & 6 spaghetti & 41 spaghetti & 147 \\
\hline
\end{tabular}




\section{Conflict of interest}

The authors declare no conflict of interest.

\section{References}

1. Ludvigsson JF, Leffler DA, Bai JC, et al. The Oslo definitions for coeliac disease and related terms. Gut 2013; 62: 43-52.

2. Sapone A, Bai JC, Ciacci C, et al. Spectrum of gluten-related disorders: consensus on new nomenclature and classification. BMJ Med 2012; 10: 13.

3. Carroccio A, Mansueto P, lacono G, et al. Non-celiac wheat sensitivity diagnosed by double-blind placebo-controlled challenge: exploring a new clinical entity. Am J Gastroenterol 2012; 107: 1898-906.

4. Elli L, Dolfini E, Bardella MT. Gliadin cytotoxicity and in vitro cell cultures. Toxicol Lett 2003; 146: 1-8.

5. Sapone A, Lammers KM, Mazzarella G, et al. Differential mucosal IL-17 expression in two gliadin-induced disorders: gluten sensitivity and the autoimmune enteropathy celiac disease. Int Arch Allergy Immunol 2010; 152: 75-80.

6. Troncone R, Jabri B. Celiac disease and gluten-sensitivity. J Intern Med 2011; 269: 582-90.

7. Thompson WG, Longstreth GF, Drossman DA, et al. Functional bowel disorders and functional abdominal pain. Gut 1999; 45: 43-7.

8. Dreborg S. Allergen standardisation and skin test. EAACI position paper. Allergy 1993; 48 (Suppl. 14): 49-82.

9. Bindslev-Jensen C, Ballmer-Weber BK, Bengtsson U, et al. Standardization of food challenges in patients with immediate reactions to foods - position paper from the European Academy of Allergology and Clinical Immunology. Allergy 2004; 59: 690-7.

10. Nowak-Wegrzyn A, Assa'ad AH, Bahna SL, et al. Adverse reactions to Food Committee of American Academy of Allergy, Asthma \& Immunology. Work Group report: oral food challenge testing. J Allergy Clin Immunol 2009; 123 (Suppl): S365-83. 